\title{
Experiencias sanitarias desde una perspectiva bioética y legal en la COVID-19*
}

\author{
Health experiences from a bioethical and legal perspective in the COVID-19 pandemic
}

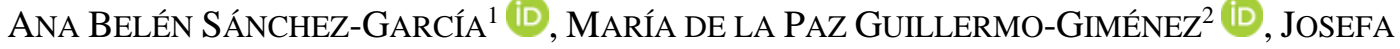 \\ MuÑOZ-SÁNCHEZ ${ }^{3}$ iD, CINDIA GÓMEZ-PÉREZ ${ }^{4}$ iD, DIEGO J. GARCÍA-CAPILlA ${ }^{5}$ (D), MARÍA \\ JOSÉ TORRALBA-MADRID ${ }^{6}$ iD
}

RESUMEN: La pandemia de la COVID-19 supone un reto para los profesionales de la salud desde un compromiso ético y moral hacia la individualidad del ser humano. El personal sanitario quedó inmerso bajo dificultades y cargas extremas haciendo imposible responder a los principios de autonomía, beneficencia, justicia, y no maleficencia. El Ministerio de Sanidad refleja la importancia de ofrecer orientaciones éticas en circunstancias excepcionales, una de estas situaciones fue priorizar la asignación de recursos humanos y materiales bajo valores éticos en la toma de decisiones con los recursos disponibles. El Comité de Bioética español propuso tener en cuenta el criterio de utilidad, equidad y protección para la no discriminación, aspectos no considerados en cuidados intensivos y en las residencias de mayores, unido a una mala planificación de recursos que produjo altos índices de mortandad. El área de cuidados paliativos se vio igualmente afectada, por lo que se aboga por un equipo de referencia para la toma de decisiones, asesoramiento, control de síntomas y malestar emocional. La vulnerabilidad patente ha promovido valores de empatía, solidaridad y compasión, también ha dejado de lado emociones, intimidad y privacidad, motivando la reflexión sobre una mayor humanización en los cuidados sanitarios. Poner en práctica el principio de justicia, el razonamiento bioético, moral y el conocimiento científico, junto a la toma de decisiones desde el respeto al paciente durante su salud y muerte, es primordial en aras a apoyar a estos profesionales en la toma de decisiones durante la crisis de la pandemia de la COVID-19.

Palabras clave: COVID-19, pandemia, ética, legal, moral, bioética, toma de decisiones.

ABSTRACT: The COVID-19 pandemic challenges health professionals with an ethical and moral commitment to the individuality of the human being. Health care workers were immersed under extreme hardships and burdens making it impossible to respond to the principles of autonomy, beneficence, justice, and non-maleficence. The Ministry of Health reflects the importance of offering ethical guidance in exceptional circumstances, one of these situations was to prioritise the allocation of human and material resources under ethical values in decision making with the available resources. The Spanish Bioethics Committee proposed taking into account the criteria of usefulness, equity and

\footnotetext{
* Fecha de recepción: 24/05/2021 - Fecha de aceptación: 03/09/2021. Cita recomendada: Sánchez-García, A. B., Guillermo-Giménez, M.P., Muñoz-Sánchez, J., Gómez-Pérez, C., García-Capilla, D. J., \& Torralba-Madrid, M. J. (2021). Experiencias sanitarias desde una perspectiva bioética y legal en la COVID-19. Bioderecho.es, (13), 1-10. https://doi.org/10.6018/bioderecho.480961

${ }^{1}$ Doctora en Enfermería por la Universidad de Murcia, Profesora asociada en la Facultad de Enfermería de la Universidad de Murcia. Correo: absg2@um.es

${ }^{2}$ Licenciada en Psicología y Pedagogía, Ayuntamiento de Murcia. Correo: mpazguillermo@ hotmail.com

${ }^{3}$ Máster en Bioderecho, Enfermera en el Servicio Murciano de Salud. Correo: pepamusa87@gmail.com

4 Máster en Urgencias y Emergencias, Enfermera en el Servicio de Salud de las Islas Baleares. Correo: cindiagomezperez@gmail.com

${ }^{5}$ Doctor en Filosofía por la Universidad de Murcia, Profesor asociado en la Facultad de Filosofía de la Universidad de Murcia. Correo:djgarcia@um.es

${ }^{6}$ Doctora en Enfermería por la Universidad Complutense de Madrid, Profesora colaboradora honoraria de la Facultad de Enfermería de la Universidad de Murcia. Correo: mjtorral@um.es
} 
protection for non-discrimination, aspects not considered in intensive care and nursing homes, together with poor resource planning, which led to high mortality rates. The area of palliative care was equally affected, which is why a referral team is advocated for decision making, counselling, symptom control and emotional distress. Patent vulnerability has promoted values of empathy, solidarity and compassion, but it has also left aside emotions, intimacy and privacy, prompting reflection on further humanisation in health care. Putting into practice the principle of justice, bioethical and moral reasoning and scientific knowledge, together with decision making based on respect for the patient during their health and death, is essential in order to support these professionals in their decision making during the crisis of the Covid 19 pandemic.

Keywords: COVID-19, pandemic, ethics, legal, moral, bioethics, decision-making.

SUMARIO: I. Introducción. II. Metodología. 1. Revisión y selección de estudios. III. Resultados. IV. DISCUSIÓN. V. CONCLUSIONES Y PROPUESTAS/REFLEXIÓN PERSONAL. VI. BIBLIOGRAFÍA. 


\section{INTRODUCCIÓN}

La COVID-19, causada por el coronavirus SAR-COV-2, fue comunicada en Wuhan (China) el 31 de diciembre de 2019, tres meses más tarde, preocupados por la rapidez de la propagación y la gravedad de la enfermedad, la OMS determinó el estado de pandemia ${ }^{1}$. Esta pandemia ha provocado una crisis económica y social, a la vez que ha supuesto un reto para los profesionales de la salud. En este contexto surgen problemas éticos que precisan de planes protocolizados con evidencia científica para su resolución y las distintas medidas adoptadas tendrían que basarse en los distintos principios bioéticos ${ }^{2}$. Además de los protocolos, se necesitan recursos materiales y más importante aún, profesionales sanitarios que realicen una correcta toma de decisiones clínicas en la atención a los pacientes, lo que implican un compromiso ético y moral ${ }^{3}$.

En 2009 durante la pandemia por el virus H1N1, surgieron necesidades éticas derivadas de la contraposición de la protección de la salud colectiva frente a los derechos individuales, la priorización de unos pacientes frente a otros, el miedo al contagio propio o a la familia, la controversia con la creación de la vacuna y el desplazamiento de la atención a otras enfermedades frente al virus de la pandemia ${ }^{4}$.

En la crisis actual, se vuelven a hacer visibles esos clásicos dilemas bioéticos, si bien la ética de la salud pública señala que las medidas que se toman deben ser lo menos intrusivas posibles puesto que, aunque haya que anteponer los problemas que afectan a la comunidad, no se debe olvidar los derechos de los individuos. En este sentido, surgen controversias con el rastreo tecnológico, que no es compatible con los principios europeos de protección de datos, y las medidas de confinamiento 5 .

El estado de alarma y confinamiento obligatorio de la población fue una medida a corto plazo que buscaba limitar la propagación del virus, mientras se intentaba adaptar el sistema sanitario a las necesidades de la crisis sanitaria ${ }^{6}$. Sin embargo, las condiciones de confinamiento marcan una diferencia entre los individuos: quedarse en casa y lavarse las manos como medidas de prevención, no son medidas que se puedan realizar de la misma manera en todas las familias por la diversidad de condiciones presentes en las mismas. Del mismo modo ha sucedido en el ámbito educativo, aunque internet ha sido un aliado en el establecimiento de relaciones, marca una distancia entre aquellas familias que tienen acceso a él y las que no ${ }^{7}$.

${ }^{1}$ ORGANIZACIÓN MUNDIAL DE LA SALUD, Cronología de la respuesta de la OMS a la COVID-19, Organización Mundial de la Salud, Ginebra, 2020. Disponible en https://www.who.int/es/news/item/29-06-2020-covidtimeline [fecha de acceso 4 de marzo de 2021].

2 ALARCÓ, A., "Aspectos éticos en una situación de pandemia", Gaceta médica, 2020. Disponible en https://gacetamedica.com/opinion/tribunas/aspectos-eticos-en-una-situacion-de-pandemia/ [fecha de acceso 4 de marzo de 2021].

${ }^{3}$ GONZÁLEZ, E., "Aspectos éticos en la toma de decisiones de COVID-19 en Atención Primaria”, AMF, Vol. 16, núm. 7, 2020. Disponible en https://amf-semfyc.com/web/article_ver.php?id=2683

${ }^{4}$ ARIAS BOHIGAS, P., "La ética durante las crisis sanitarias: a propósito de la pandemia por el virus H1N1", Revista Española de Salud Pública, Vol. 83, núm. 4, 2009, pp. 489-491. Disponible en http://scielo.isciii.es/scielo.php?script=sci_arttext\&pid=S1135-57272009000400001

${ }^{5}$ CASADO, M., Dilemas bioéticos de la pandemia [vídeo], Observatori de Bioètica i Dret de la Universitat de Barcelona, Barcelona, 2020. Disponible en https://www.youtube.com/watch?v=J0kJDRpzD8o

${ }^{6}$ CUESTAS, M., MINASSIAN, M., “COVID-19: Ecos de una pandemia”, Revista Argentina de Microbiología, Vol. 52, núm. 3, 2020, pp. 167-168. https://doi.org/10.1016/j.ram.2020.09.003

${ }^{7}$ CASADO, M., Dilemas bioéticos..., op. cit. 
Además, durante el pico de la pandemia en la primera ola el personal sanitario se enfrentó a una serie de dificultades, entre ellas la falta de equipos de protección personal (EPI), el triaje de pacientes candidatos a acceder a cuidados intensivos debido a la escasez de recursos necesarios, la falta de personal que suponía cargas extremas de trabajo, el estrés familiar y personal de esta situación, entre otras características. Los recursos de Atención Primaria se centraron en el virus dejando de atender a pacientes crónicos, pacientes con patologías agudas y graves que no llegaban a consultar por dificultad de acceso al sistema sanitario o por miedo al contagio. Viéndose en estos casos afectados los principios de autonomía, beneficencia, justicia y no maleficiencia ${ }^{8}$. También surgió un conflicto con las personas mayores institucionalizadas en las residencias, dependiendo de la zona geográfica, podían o no acceder a los centros de salud u hospitales, además de la muerte en soledad que muchos mayores sufrieron ${ }^{9}$. Por tanto, en esta crisis sanitaria la bioética debe intentar sugerir soluciones que se anticipen y minimicen las consecuencias negativas de la toma de decisiones ${ }^{10}$.

Los objetivos planteados son conocer las experiencias sanitarias desde una perspectiva bioética y legal en la COVID-19.

\section{METODOLOGÍA}

\section{Revisión y selección de estudios}

Se realizó una búsqueda bibliográfica en diferentes bases de datos: Science Direct, Cuiden y web del Ministerio de Sanidad, Consumo y Bienestar Social. Se utilizaron los siguientes descriptores Mesh y Decs: "COVID-19", "pandemia", "ética", "legal", "moral", "bioética", "toma de decisiones", formulando combinaciones con los operadores boleanos "AND (Y)/OR (O)”.

Los artículos seleccionados tras lectura de título y resumen fueron tanto en castellano como en inglés, analizando a texto completo los que se ajustaban a los objetivos de la investigación, añadiendo también artículos por búsqueda inversa. La temporalidad de los mismos fue de marzo-diciembre 2020.

\section{RESULTADOS}

Santillán-García refleja cómo los aspectos morales, bioéticos y científicos influyen y guían en la toma de decisiones en el contexto de la pandemia por COVID-19, incluyendo la sensibilidad moral, el razonamiento bioético y el conocimiento en toma de decisiones clínicas como aspectos fundamentales en situaciones de escasez y asignación de recursos ${ }^{11}$.

\footnotetext{
${ }^{8}$ GONZÁLEZ, E., “Aspectos éticos...”, op. cit.; CABRÉ, L., Criterios clínicos para el triaje de pacientes críticos en situación de emergencia [video], Observatori de Bioètica i Dret de la Universitat de Barcelona, Barcelona, 2020. Disponible en https://www.youtube.com/watch?v=lv0xpwMZxDs

${ }^{9}$ RODRÍGUEZ, P., Conflictos de la pandemia en residencias, sociosanitarios y servicios sociales [video], Observatori de Bioètica i Dret de la Universitat de Barcelona, Barcelona, 2020. Disponible en https://www.youtube.com/watch?v=VHe6GBk550k

${ }^{10}$ CASADO, M., "Dilemas bioéticos...", op. cit.

11 SANTILlÁN-GARCÍA, A., FERRER-ARNEDO, C., "Consideraciones éticas durante las crisis sanitarias: a propósito de la pandemia por el coronavirus SARS-CoV-2", Revista Española de Salud Pública, Vol. 94, 2020, p. 1-8. https://www.mscbs.gob.es/biblioPublic/publicaciones/recursos_propios/resp/revista_cdrom/VOL94/C_ESPECIALES /RS94C_202011149.pdf
} 
En este sentido también Coronado anima a analizar diferentes aspectos éticos que han generado debate bioético durante la pandemia, recordando a su vez los dilemas que surgieron en la pandemia por el $\mathrm{N} 1 \mathrm{H} 1$, lo que permite reflexionar si la sociedad ha aprendido en la actual crisis sanitaria, sin embargo, no ha sido lo suficiente, para el abordaje de esta situación es necesario hacerlo con las mejores evidencias y basándose en los principios éticos básicos ${ }^{12}$. Así como conocer las debilidades que han conducido a una tasa de mortalidad elevada por COVID-19 y analizar sus consecuencias con el objetivo de proporcionar propuestas de mejora, entre ellas, destacar cómo la pandemia ha dificultado la atención de los pacientes en situación de final de vida y la necesidad de incorporar un equipo de cuidados paliativos en las próximas unidades COVID $^{13}$.

A su vez Sarrión refleja cómo las competencias de las autoridades sanitarias para restringir derechos en situación de crisis sanitaria explora el fundamento constitucional de la protección a la salud y la competencia de las autoridades sanitarias para restringir o limitar los derechos de los ciudadanos en la lucha contra la pandemia ${ }^{14}$. En la práctica clínica las experiencias sanitarias llevan a una reflexión sobre el papel de las cuestiones éticas con las que se han encontrado los profesionales dedicados a los cuidados críticos, tal y como afirma Yepes-Temiño, ante la escasez de recursos sanitarios y un triaje en el que los mayores no tuvieron acceso a un respirador ${ }^{15}$. De esta forma Casas Martínez aporta como enseñanzas de la COVID-19 el reencuentro con la vulnerabilidad humana, lo que permite tomar conciencia sobre cómo la actual pandemia, un escenario fuera del control de la persona, hace visible la vulnerabilidad humana y cómo la formación moral y su detonante emotivo pueden ser útiles para desarrollar estrategias eficaces en futuras emergencias ${ }^{16}$.

\section{DISCUSIÓN}

Una crisis sanitaria activa los deberes morales, legales y científicos en la labor de proteger el derecho a la salud y a la vida. La pandemia ha puesto en evidencia que nuestros recursos son limitados durante tiempos de "urgencia" y ha generado dilemas éticos y conflictos de interés en la atención, entre la ética de la salud pública y la ética clínica ${ }^{17}$.

La Salud Pública, cuyo objetivo es cuidar y mejorar la salud de la población y de las personas que forman parte de ella, durante momentos de crisis sanitaria se rige por el principio de justicia social (justicia distributiva), este principio difiere de la ética clínica, puesto que da

12 CORONADO-VÁZQUEZ, V., CASTRO-MARTÍN, J., CÁMARA-ESCRIBANO, C., GÓMEZ-SALGADO, J., MARTÍN-LÓPEZ, C., GARCÍA-IGLESIAS, J. J., "Dimensiones éticas de la prevención y planificación en los centros sociosanitarios durante la pandemia por SARS-CoV-2 (Covid-19): una emergencia de salud pública", Revista Española de Salud Pública, Vol. 94, 2020, $\quad$ pp. $1-8 . \quad$ Disponible en https://www.mscbs.gob.es/biblioPublic/publicaciones/recursos_propios/resp/revista_cdrom/VOL94/C_ESPECIALES /RS94C_202009105.pdf

13 Ibídem.

${ }^{14}$ SARRIÓN ESTEVE, J., "La competencia de las autoridades sanitarias para restringir derechos en situación de crisis sanitaria”, Gaceta Sanitaria, 2020. https://doi.org/10.1016/j.gaceta.2020.07.009

${ }^{15}$ YEPES-TEMIÑO M. J., CALLEJAS GONZÁLEZ, R., ÁLVAREZ AVELLO, J. M., "Un momento para la reflexión. Aspectos éticos en la pandemia SARS-CoV- 2/COVID-19 en nuestra práctica clínica", Revista Española de Anestesiología y Reanimación, Vol. 68, núm. 1, 2021, pp. 28-36. https://doi.org/10.1016/j.redar.2020.09.004

${ }^{16}$ CASAS MARTÍNEZ, M. L., "Enseñanzas de la pandemia COVID-19. El reencuentro con la vulnerabilidad humana", Bioethics UPdate, Vol. 6, núm. 2, 2020, pp. 80-91. https://doi.org/10.1016/j.bioet.2020.09.001

${ }^{17}$ CORONADO-VÁZQUEZ, V., CASTRO-MARTÍN, J., CÁMARA-ESCRIBANO, C., GÓMEZ-SALGADO, J., MARTÍN-LÓPEZ, C., GARCÍA-IGLESIAS, J. J., "Dimensiones éticas...," op. cit.; CAMARGO RUBIO, R. D., "Los aspectos morales bioéticos y científicos guían las decisiones en el contexto de recursos escasos durante la pandemia por COVID-19”, Acta Colombiana de Cuidado Intensivo, 2020. https://doi.org/10.1016/j.acci.2020.10.005 
prioridad al bien común por encima de la autonomía individual ${ }^{18}$. Es ahí donde surge un conflicto entre la limitación de los derechos individuales de cada persona frente a la protección de la salud de la sociedad. Durante la pandemia por COVID-19, los ciudadanos están viviendo restricciones de movilidad o cuarentena en la lucha contra el virus ${ }^{19}$. Ante esta situación, existe fundamento constitucional y cobertura legal. El art. 43 de la Constitución Española les confiere a los poderes públicos la competencia para organizar y tutelar la salud pública y en el art. 32.4 se indica que mediante ley se pueden regular los deberes en casos de riesgo grave, catástrofe o calamidad pública. También el art. 54 de la Ley 33/2011, de 4 de octubre, General de Salud Pública (LGSP) contempla que las autoridades competentes podrán adoptar medidas de inmovilización, cierre preventivo, suspensión de actividades y otras medidas si existen indicios de razones de riesgo para la salud. Además, en la Ley 41/2002, de 14 de noviembre, básica reguladora de la autonomía del paciente y de derechos y obligaciones en materia de información y documentación clínica, el art. 9.2 considera la intervención clínica en favor de la salud del paciente, sin necesidad de su consentimiento, cuando existe un riesgo ${ }^{20}$.

Ahora bien, como se ha mencionado anteriormente, la ética clínica, en favor de la autonomía, no se deja de lado, sino que se debe actuar junto con el principio de justicia distributiva y se debe complementar además con otros principios como el de igualdad, equidad, transparencia y dignidad. Puesto que no se podría entender la justicia en una situación de inequidad, ni sin los demás principios éticos que la respalden ${ }^{21}$.

Si bien se han publicado trabajos sobre posibles escenarios en otras pandemias de la historia, con posibles medidas y principios de actuación, no ha sido lo suficientemente efectivo, esto puede ser debido a que, como señala Yepes-Temiño se han quedado en hipótesis ${ }^{22}$ de actuación o porque las cifras de esta pandemia han superado las expectativas ${ }^{23}$. Motivo por el que desde el inicio de la pandemia se hicieron necesarias propuestas sobre aspectos éticos, difundidas por sociedades sanitarias especializadas que estaban tratando de paliar el problema creciente en primera línea antes que por expertos a nivel nacional.

Situación alarmante en la sociedad consecuencia de establecer criterios y protocolos que no seguían unos criterios comunes, sino que cada centro o sociedad médica lanzaba sus criterios bioéticos, dejando a la luz una falta de uniformidad con grandes diferencias de decisiones clínicas entre los diferentes centros sanitarios. De esta forma el Ministerio de Sanidad, en su informe sobre los aspectos éticos, destaca que durante la crisis sanitaria es importante ofrecer orientaciones éticas para circunstancias excepcionales ${ }^{24}$.

En este sentido, una de las situaciones en las que fue primordial tener un marco de referencia ético para la toma de decisiones fue priorizar la asignación de recursos. Como apunta Camargo, la gran demanda de tratamientos hospitalarios junto con la falta de recursos humanos y materiales como equipos de protección personal (EPI) hizo necesario establecer un criterio

\footnotetext{
${ }^{18}$ CAMARGO RUBIO, R. D., "Los aspectos morales bioéticos...”, op. cit.

${ }^{19}$ SANTILLÁN-GARCÍA, A., FERRER-ARNEDO, C., “Consideraciones éticas...”, op. cit.

${ }^{20}$ SARRIÓN ESTEVE, J., "La competencia de las autoridades...", op. cit.

${ }^{21}$ CAMARGO RUBIO, R. D., "Los aspectos morales bioéticos...”, op. cit.; CORONADO-VÁZQUEZ, V., CASTROMARTÍN, J., CÁMARA-ESCRIBANO, C., GÓMEZ-SALGADO, J., MARTÍN-LÓPEZ, C., GARCÍA-IGLESIAS, J. J., "Dimensiones éticas...", op. cit.

22 YEPES-TEMIÑO M. J., CALLEJAS GONZÁlEZ, R., ÁlVAREZ AVELlO, J. M., "Un momento para la reflexión...", op. cit.

${ }^{23}$ SARRIÓN ESTEVE, J., "La competencia de las autoridades...," op. cit.

24 YEPES-TEMIÑO M. J., CALLEJAS GONZÁlEZ, R., ÁlVAREZ AVELlO, J. M., "Un momento para la reflexión...", op. cit.
} 
avalado por múltiples valores éticos que se adaptasen de forma individual en la toma de decisión a la hora de asignar esos recursos limitados a determinados pacientes ${ }^{25}$. El Comité de Bioética español propuso establecer un modelo mixto, en el que se tuviera en cuenta tanto el criterio de utilidad, equidad y el de protección frente a vulnerabilidad, para garantizar que las personas no sean discriminadas en una decisión ${ }^{26}$.

Esta priorización y sus casos más sobrecogedores se han visto sobre todo en cuidados intensivos en relación con las plazas y el acceso a ventilación mecánica, donde se generó la obligación de seleccionar a aquellos pacientes que podía obtener más beneficios ${ }^{27}$. Diariamente los profesionales realizan toma de decisiones fundamentándose en criterios clínicos basados en la evidencia y recomendaciones de sociedades científicas ${ }^{28}$, pero en esta situación de pandemia se ha visto necesario un cambio en la connotación del triaje, realizándose de forma similar a las situaciones de medicina de catástrofes, basándose en la justicia distributiva y la asignación adecuada de recursos. A la hora de aplicar el triaje no se debe basar en un único criterio y ante un paciente con vulnerabilidades, los factores como la edad, comorbilidad o discapacidad no deben ser discriminatorios para la toma de decisiones, priorizándose los criterios médicos a los pacientes con mayor posibilidad clínica de recuperarse ${ }^{29}$.

En relación con esa afirmación, durante el pico de la pandemia, la selección de pacientes con mayor probabilidad de beneficiarse repercutió directamente en las personas mayores de residencias y pacientes de centros sociosanitarios. La Ley 39/2006, de 14 de diciembre, de Promoción de la Autonomía Personal y Atención a las Personas en Situación, establece los procedimientos que garanticen la cohesión y equidad territorial en la asistencia integral de persona dependientes. Y en los meses de mayor tensión, los factores anteriormente mencionados fueron clave y la mala planificación de recursos conllevó la gran cantidad de muertes por COVID-19 en personas de estos centros. Por lo tanto, un aspecto importante a la hora de la aplicación de dichos protocolos es la ética de la transparencia, que garantiza que no hay decisiones arbitrarias y evidencia respeto a la dignidad de las personas y compromiso con el bien común ${ }^{30}$.

Otro aspecto para destacar es el de aquellos pacientes que por razones científicas no han podido ser beneficiados de esos recursos, deberían llevar una muerte digna por medio de los cuidados paliativos ${ }^{11}$. Sin embargo, este ámbito asistencial también se ha visto afectado, en el sentido que, no todos los profesionales que han manejado medicamentos para situaciones de final de vida tenían una suficiente formación, puesto que la gran cantidad de pacientes y la falta de personal, han llevado a la creación de "guías de tratamiento rápido" que no se adaptan a las situaciones individuales del paciente y su pronóstico, o la ausencia de contacto con familiares e incluso con los propios profesionales por posibilidad de contagio y falta de equipos de protección. Por ello, se aboga por la inclusión de un equipo cuidados paliativos de referencia para la toma de

\footnotetext{
${ }^{25}$ CAMARGO RUBIO, R. D., "Los aspectos morales bioéticos...”, op. cit.

${ }^{26}$ Ibídem; YEPES-TEMIÑO M. J., CALLEJAS GONZÁLEZ, R., ÁLVAREZ AVELLO, J. M., "Un momento para la reflexión...", op. cit.

${ }^{27}$ CORONADO-VÁZQUEZ, V., CASTRO-MARTÍN, J., CÁMARA-ESCRIBANO, C., GÓMEZ-SALGADO, J., MARTÍN-LÓPEZ, C., GARCÍA-IGLESIAS, J. J., "Dimensiones éticas...”, op. cit.

${ }^{28}$ SANTILLÁN-GARCÍA, A., FERRER-ARNEDO, C., "Consideraciones éticas...”, op. cit.

29 YEPES-TEMIÑO M. J., CALLEJAS GONZÁlEZ, R., ÁlVAREZ AVELlO, J. M., "Un momento para la reflexión...", op. cit.; CAMARGO RUBIO, R. D., "Los aspectos morales bioéticos...”, op. cit.

${ }^{30}$ Ibídem.
} 
decisiones, asesoramiento y supervisión del control de síntomas, así como para atender el malestar emocional $^{31}$.

Y por último mencionar que la pandemia por COVID-19 ha dejado expuesta la vulnerabilidad humana y ésta al ser compartida se ha visto cómo puede promover valores como la empatía, la solidaridad, compasión, la ayuda... ${ }^{32}$. Pero a la vez, la atención se centraba en el valor de la vida, dejando de lado las emociones y su cuidado, la intimidad, la privacidad, entre otros $^{33}$. Por ello, se necesitaría de una humanización en la atención, lo cual sería un buen punto para reflexionar en futuras situaciones de emergencia.

En cuanto a las limitaciones que se pueden encontrar en los artículos incluidos estarían relacionadas con la evolución de la pandemia, puesto que la mayoría de los artículos son reflexiones sobre diversos dilemas éticos o morales que surgieron durante el pico de la pandemia, por lo que no reflejan la situación que puede estar aconteciendo mientras se escribe el presente trabajo. Otra limitación sería que los trabajos se centran sobre todo en el aspecto bioético y moral pero no aluden a implicaciones legales.

Refiriéndose a las limitaciones de la revisión realizada encontramos que, si bien describe dichos conflictos, se necesitarían conocer de manera más detallada para lo cual sería necesario centrarse en uno de ellos. Además, la revisión recoge artículos que se refieren a situaciones en el ámbito hospitalario, aludiendo brevemente también a los centros sociosanitarios y residencias, pero sin tener en cuenta por ejemplo el ámbito de Atención Primaria, donde se plantean distintos problemas éticos.

\section{CONCLUSIONES Y PROPUESTAS/REFLEXIÓN PERSONAL}

La crisis sanitaria por la COVID-19 ha generado diversos dilemas éticos a la hora de atender y cuidar de los pacientes y en la sociedad en general, donde la perspectiva moral y legal pone de manifiesto los conflictos sanitarios ante la escasez de recursos materiales y humanos con un triaje que impide la vida a los mayores entre otros colectivos.

Es fundamental el principio de justicia distributiva que rige la Salud Pública junto con otros razonamientos bioéticos, morales y conocimiento científico que han sido fundamentales en la toma de decisiones durante la escasez de recursos para respetar los derechos y la dignidad de los pacientes durante su salud y muerte.

Mejorar los protocolos de actuación sanitaria es primordial desde una perspectiva bioética ya que son los que apoyan a los profesionales en la solución de problemas éticos que puedan surgir durante una crisis sanitaria y ayudan a disminuir el gran impacto emocional que una pandemia genera en la sociedad actual.

\section{BIBLIOGRAFÍA}

- ALARCÓ, A., "Aspectos éticos en una situación de pandemia”, Gaceta médica, 2020. Disponible en https://gacetamedica.com/opinion/tribunas/aspectos-eticos-en-una-situacionde-pandemia/ [fecha de acceso 4 de marzo de 2021].

\footnotetext{
${ }^{31}$ CORONADO-VÁZQUEZ, V., CASTRO-MARTÍN, J., CÁMARA-ESCRIBANO, C., GÓMEZ-SALGADO, J., MARTÍN-LÓPEZ, C., GARCÍA-IGLESIAS, J. J., “Dimensiones éticas...”, op. cit.

${ }^{32}$ CASAS MARTÍNEZ, M. L., "Enseñanzas de la pandemia COVID-19. El reencuentro con la vulnerabilidad humana", Bioethics UPdate, Vol. 6, núm. 2, 2020, pp. 80-91. https://doi.org/10.1016/j.bioet.2020.09.001

${ }^{33}$ SANTILLÁN-GARCÍA, A., FERRER-ARNEDO, C., "Consideraciones éticas...”, op. cit.
} 
- ARIAS BOHIGAS, P., "La ética durante las crisis sanitarias: a propósito de la pandemia por el virus H1N1", Revista Española de Salud Pública, Vol. 83, núm. 4, 2009, pp. 489-491. Disponible en $\quad \underline{\mathrm{http}} / / / \mathrm{scielo}$.isciii.es/scielo.php?script=sci $\operatorname{arttext\& pid=S1135-}$ 57272009000400001.

- CABRÉ, L., Criterios clínicos para el triaje de pacientes críticos en situación de emergencia [video], Observatori de Bioètica i Dret de la Universitat de Barcelona, Barcelona, 2020. Disponible en https://www.youtube.com/watch?v=lv0xpwMZxDs

- CAMARGO RUBIO, R. D., "Los aspectos morales bioéticos y científicos guían las decisiones en el contexto de recursos escasos durante la pandemia por COVID-19", Acta Colombiana de Cuidado Intensivo, 2020. https://doi.org/10.1016/j.acci.2020.10.005

- CASADO, M., Dilemas bioéticos de la pandemia [vídeo], Observatori de Bioètica i Dret de la Universitat de Barcelona, Barcelona, 2020. Disponible en https://www.youtube.com/watch?v=J0kJDRpzD80

- CASAS MARTÍNEZ, M. L., "Enseñanzas de la pandemia COVID-19. El reencuentro con la vulnerabilidad humana", Bioethics UPdate, Vol. 6, núm. 2, 2020, pp. 80-91. https://doi.org/10.1016/j.bioet.2020.09.001

- CORONADO-VÁZQUEZ, V., CASTRO-MARTÍN, J., CÁMARA-ESCRIBANO, C., GÓMEZ-SALGADO, J., MARTÍN-LÓPEZ, C., GARCÍA-IGLESIAS, J. J., "Dimensiones éticas de la prevención y planificación en los centros sociosanitarios durante la pandemia por SARS-CoV-2 (Covid-19): una emergencia de salud pública", Revista Española de Salud Pública, Vol. 94, 2020, pp. 1-8. Disponible en https://www.mscbs.gob.es/biblioPublic/publicaciones/recursos_propios/resp/revista cdrom/ VOL94/C_ESPECIALES/RS94C_202009105.pdf

- CUESTAS, M., MINASSIAN, M., "COVID-19: Ecos de una pandemia”, Revista Argentina de Microbiología, Vol. 52, núm. 3, 2020, pp. 167-168. https://doi.org/10.1016/j.ram.2020.09.003

- GONZÁlEZ, E., "Aspectos éticos en la toma de decisiones de COVID-19 en Atención Primaria", $A M F$, Vol. 16, núm. 7, 2020. Disponible en https://amfsemfyc.com/web/article_ver.php?id=2683

- ORGANIZACIÓN MUNDIAL DE LA SALUD, Cronología de la respuesta de la OMS a la COVID-19, Organización Mundial de la Salud, Ginebra, 2020. Disponible en https://www.who.int/es/news/item/29-06-2020-covidtimeline [fecha de acceso 4 de marzo de 2021].

- RODRÍGUEZ, P., Conflictos de la pandemia en residencias, sociosanitarios y servicios sociales [video], Observatori de Bioètica i Dret de la Universitat de Barcelona, Barcelona, 2020. Disponible en https://www.youtube.com/watch?v=VHe6GBk550k

- SANTILlÁN-GARCÍA, A., FERRER-ARNEDO, C., "Consideraciones éticas durante las crisis sanitarias: a propósito de la pandemia por el coronavirus SARS-CoV-2", Revista Española de Salud Pública, Vol. 94, 2020, pp. 1-8. https://www.mscbs.gob.es/biblioPublic/publicaciones/recursos_propios/resp/revista_cdrom/ VOL94/C ESPECIALES/RS94C 202011149.pdf

- SARRIÓN ESTEVE, J., "La competencia de las autoridades sanitarias para restringir derechos en situación de crisis sanitaria", Gaceta Sanitaria, 2020. https://doi.org/10.1016/j.gaceta.2020.07.009 
- YEPES-TEMIÑO M. J., CALlEJAS GONZÁlEZ, R., ÁlVAREZ AVELLO, J. M., "Un momento para la reflexión. Aspectos éticos en la pandemia SARS-CoV- 2/COVID-19 en nuestra práctica clínica", Revista Española de Anestesiología y Reanimación, Vol. 68, núm. 1, 2021, pp. 28-36. https://doi.org/10.1016/j.redar.2020.09.004 